\title{
Variation in United States COVID-19 newborn care practices: results of an online physician survey
}

\author{
Margaret G. Parker ${ }^{1 *}$, Arun Gupta ${ }^{2}$, Helen Healy ${ }^{3}$, Aviel Peaceman' ${ }^{1}$ Stephen M. Kerr ${ }^{4}$, Timothy C. Heeren ${ }^{5}$,
} Mark L. Hudak ${ }^{6}$ and Munish Gupta ${ }^{7}$

\begin{abstract}
Background: Newborn care practices that best promote the health and well-being of mother-infant dyads after birth while minimizing transmission of COVID-19 were uncertain at the onset of the COVID-19 pandemic.

Objective: Examine variation in COVID-19 newborn care practices among U.S. birth hospitals and by hospital characteristics (U.S. census region, highest level of neonatal level of care, and Baby-Friendly hospital status).

Study Design: We surveyed physicians via American Academy of Pediatrics email listservs and social media between 5/26/2020-6/8/2020. Physicians identified the birth hospital in which they provided newborn care and their hospital's approach to obstetrical and newborn care related to COVID-19. Chi-square tests were used to examine variation in hospital practices by U.S. census region, highest level of neonatal care, and Baby-Friendly hospital status.

Results: Four hundred thirty three physicians responded from 318 hospitals across 46 states. Variation in care of SARS-CoV-2 positive mother-infant dyads was greatest for approaches to location of newborn care (31\% separation, $17 \%$ rooming-in, and $51 \%$ based on shared-decision making), early skin-to-skin care (48\% prohibited/discouraged, $11 \%$ encouraged, and $40 \%$ based on shared-decision making) and direct breastfeeding (37\% prohibited/discouraged, 15\% encouraged, and $48 \%$ based on shared-decision making). Among presumed uninfected dyads, 59\% of hospitals discharged at least some mother-infant dyads early. We found variation in practices by U.S. census region.
\end{abstract}

Conclusion: Approaches to newborn care and breastfeeding support for mother-infant dyads with positive SARSCoV-2 testing differed across U.S. birth hospitals during the COVID-19 pandemic. Early discharge of presumed uninfected mother-infant dyads was common.

Keywords: COVID-19, Newborn care, Hospital care, Breastfeeding

\section{Introduction}

In response to the increase of pregnant women who tested positive for SARS-CoV-2 presenting to birth hospitals for delivery within the setting of the COVID-19 pandemic in the spring of 2020, U.S. hospitals rapidly

\footnotetext{
*Correspondence: margaret.parker@bmc.org

1 Department of Pediatrics, Boston Medical Center, Boston University School of Medicine, 801 Albany St, Room 2010, Boston, MA 02446, USA Full list of author information is available at the end of the article
}

created and implemented clinical guidelines pertaining to maternal and newborn peripartum care. Large, population-level studies regarding risk of mother-toinfant transmission of SARS-CoV-2 and clinical manifestations of neonatal COVID-19 disease were lacking at this time, and are more recently emerging. In the context of this uncertainty, professional organizations issued different guidance in key areas of newborn care, which led to confusion and controversy $[1,2]$. For example, on March 18, 2020, the World Health Organization 
(WHO) recommended that infants remain in close contact with SARS-CoV-2 positive mothers and directly breastfeed after delivery, if mothers are stable to do so [3], while the American Academy of Pediatrics (AAP) interim guidance from April 2, 2020 [4] and May 21, 2020 [5] recommended temporary separation of mother and infant as the safest course of action to minimize the risk of mother-to-infant transmission during the postpartum period. In comparison, guidance from the Centers for Disease Control (CDC) on April 4, 2020 [6] recommended that decisions about location of care "should be made on a case-by-case basis using shared decisionmaking between the mother and the clinical team." The language was slightly modified on May 20, 2020 [7], to "risks and benefits of temporary separation of the mother and her baby should be discussed with the mother by the healthcare team, and decisions about temporary separation should be made in accordance with the mother's wishes."

The impact of these varying recommendations on clinical practice among U.S. birth hospitals is unknown. It is unclear whether hospitals may have preferentially adopted practices aligned with the WHO, AAP, CDC, other professional organizations [8], or other practices that were possibly tailored to their local context. Further, while extensive practice changes have undoubtedly occurred that focused on mother-infant dyads with positive SARS-CoV-2 testing, the extent to which hospitals may have changed practice for dyads without positive SARS-CoV-2 testing is unknown. Finally, whether hospital-level factors may have influenced what guidance a hospital adopted has not yet been examined.

In the context of these knowledge gaps, the primary purpose of our study was to examine variation in COVID-19 related obstetrical and newborn care practices among U.S. birth hospitals. Our secondary purpose was to examine variation in COVID-19 related obstetrical and newborn care practices according to hospitallevel factors, including U.S. census region, highest level of neonatal care, and Baby-Friendly hospital status.

\section{Methods}

\section{Population and setting}

We ascertained COVID-19 newborn care practices at the hospital-level in a two-step process. First, we conducted a 5-7 minute, online, anonymous REDCap survey from May 26 to June 8, 2020 of individual physicians that provided newborn care at U.S. birth hospitals in the U.S. Because our goal was to distribute to a wide, national group of newborn care providers, we distributed the survey via email listservs of the AAP Sections on Hospital Medicine, Breastfeeding Medicine, and Neonatal Perinatal Medicine, as well as the Neonatal Physician Mothers
Facebook group and on the Twitter feeds of the authors. We asked physicians to provide responses regarding hospital-level newborn care practices and guidelines during the week of May 17 to 24, 2020 at the hospital they "work at the most." Second, we sorted the responses among individuals by the self-reported U.S. birth hospital that they work at most. If there was more than one response per hospital and the responses were the same, those responses constituted the responses for that hospital. If there was more than one response per hospital and the responses varied, we ascertained the name(s) and email address (es) of the newborn or neonatology physician medical director(s) at that hospital through personal email contacts of the authors, internet searches, and phone calls to hospitals, and clarified any discrepant responses. Specifically, we presented the survey question and answers verbatim along with the discrepant responses and asked for clarification regarding the most accurate response pertaining to their hospital's approach of care from May 17 to 24, 2020. We incorporated the final "reconciled" responses by medical directors in the final data set of newborn care practices at the hospitallevel. We chose this two-step, pragmatic approach to ascertain national COVID-19 newborn care practices to necessitate rapid feedback. This study was approved as an exempt study by the Boston University Medical Campus Institutional Review Board.

\section{Hospital Care Practices}

We chose to examine obstetric and newborn care practices that were included in professional guidance by the WHO, AAP, and CDC, as well as topics that frequently arose in the Massachusetts statewide perinatal COVID19 webinar series [9] and other similar national webinars [10]. We adapted survey questions from two previous Massachusetts statewide COVID-19 newborn care practice surveys administered to $>20$ hospital teams in April and May 2020 [9]. Our survey is included as an Appendix. We asked physicians to respond regarding hospitallevel practices of interest, including testing of mothers, infants, and support persons, visitation, personal protective equipment (PPE) at delivery, location of care (separate rooms vs. rooming-in), skin-to-skin care in the first hour after birth, early baths, delayed cord clamping, direct breastfeeding, and discharge processes and timing.

\section{Hospital Characteristics}

We examined responses by US region according to the US Census tract (Northeast, Midwest, South, and West) and highest level of neonatal care provided at the hospital $(1,2,3$, or 4$)$ according to the AAP Guidelines for Perinatal Care [11]. We included definitions of levels of neonatal care in the survey for reference. We also examined 
responses according to the hospital's Baby-Friendly status, which was obtained from the USA Baby-Friendly website [12].

\section{Individual Preference for Location of Care}

In addition to hospital-level practices, we also examined individuals' own preferred approach for location of care because this was a controversial practice at the time of survey distribution and professional organization guidance on this practice varied. We categorized responses as preferences for separation, rooming-in with precautions, or rooming-in without precautions; shared decision making on a case-by-case basis; no particular opinion; and other.

\section{Statistical Analysis}

We examined prevalence and 95\% confidence intervals (CIs) of hospital practices in our overall sample and then compared by US region, highest level of neonatal care, and Baby-Friendly hospital status, using chi-square tests. We then examined the extent to which individuals' personal preferences for location of care agreed with their hospital's recommended practice, using chi-square tests. All analyses were conducted using SAS 9.4.

\section{Results}

A flow chart of participant and hospital inclusion criteria is shown in Figure 1. Among 458 total individual respondents, we excluded 16 that did not report working at a birth hospital, 1 that reported more than one birth hospital that they worked at "the most," and 2 from nonU.S. states, leaving 439 individual respondents representing 320 unique birth hospitals in 46 states. Of these, 246 hospitals were represented by a single respondent, and 74 hospitals were represented by multiple respondents. The median and range of multiple respondents was 2 and 2 to 9, respectively. Among hospitals with multiple respondents, we excluded 2 due to lack of response from clinical/ medical directors to reconcile discrepant answers. Our final sample consisted of 318 birth hospitals, comprised of 433 individual respondents for analysis. This represents approximately $11 \%$ of current US birthing hospitals.

Overall distribution of COVID-19 related hospital care practices are shown in Table 1 . Nationally, $74 \%$ of

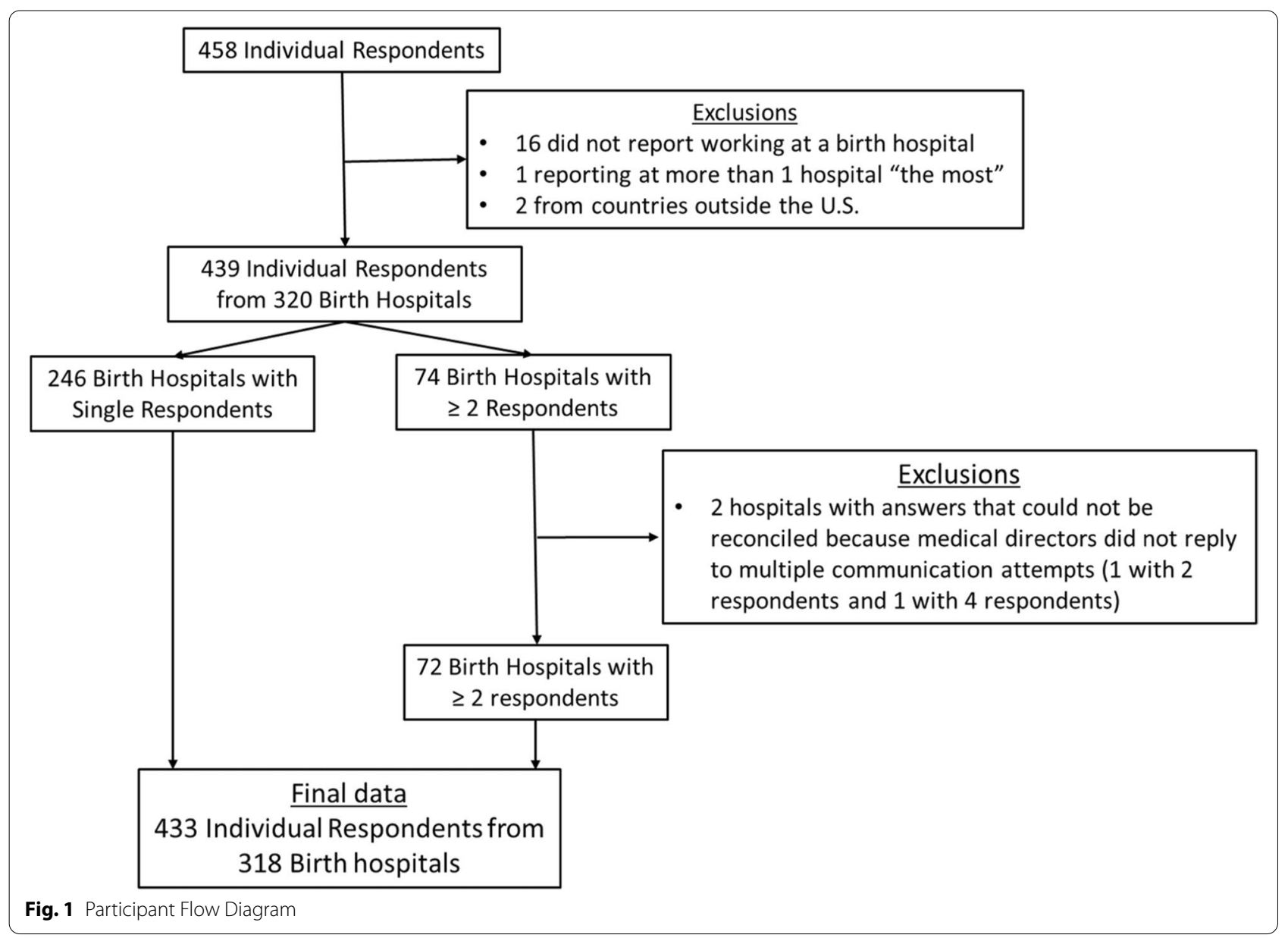


Table 1 COVID-19 Related Hospital Care Practices Among Mothers with Healthy, Term Infants Among US Birth Hospitals

\begin{tabular}{l} 
Overall, $\mathbf{n}$ (\%) \\
\hline Obstetric and Delivery Practices \\
Testing of Women Anticipated to Deliver \\
Universal testing \\
Testing based on signs and symptoms \\
Testing not routinely available for pregnant women \\
Other ${ }^{1}$ \\
PPE for COVID-19 positive women delivering vaginally (check all that apply) \\
N95 \\
Regular Surgical Mask \\
Eye protection \\
Cap \\
Gown \\
Gloves \\
Support persons for pregnant women on Labor and Delivery \\
No support persons \\
Only 1 support person \\
2 or more support persons
\end{tabular}

Approach to testing asymptomatic support persons of COVID positive mothers Not offered or required

Offer some testing

Required

$\mathrm{N}(\%)$

$95 \% \mathrm{Cl}$

$318(100 \%)$

\section{Newborn Care Practices}

Location of newborn care with COVID-19 Positive Mother

Separate room from mother

Same room as mother with precautions to maintain separation

Same room as mother with no precautions

Decisions based on shared decision making on a case-by-case basis

Other $^{2}$

Skin-to-skin care in first hour after birth with COVID-19 Positive Mother

Prohibited

Discouraged

Encouraged with precautions

Encouraged with no precautions

Decisions based on shared decision making on a case-by-case basis

\begin{tabular}{|c|c|}
\hline 235 (73.9\%) & $68.7 \%, 78.6 \%$ \\
\hline 68 (21.4\%) & $17.0 \%, 26.3 \%$ \\
\hline $3(0.9 \%)$ & $0.2 \%, 2.7 \%$ \\
\hline 10 (3.1\%) & $1.5 \%, 5.7 \%$ \\
\hline 305 (95.9\%) & $93.1 \%, 97.8 \%$ \\
\hline 17 (5.3\%) & $3.1 \%, 8.4 \%$ \\
\hline 310 (97.5\%) & $95.1 \%, 98.9 \%$ \\
\hline 206 (64.8\%) & $59.3 \%, 70.0 \%$ \\
\hline 305 (95.9\%) & $93.1 \%, 97.8 \%$ \\
\hline 310 (97.5\%) & $95.1 \%, 98.9 \%$ \\
\hline $2(0.6 \%)$ & $0.1 \%, 2.3 \%$ \\
\hline 311 (97.8\%) & $95.5 \%, 99.1 \%$ \\
\hline $3(0.9 \%)$ & $0.2 \%, 2.7 \%$ \\
\hline 250 (78.6\%) & $73.7 \%, 83.0 \%$ \\
\hline 41 (12.9\%) & $9.4 \%, 17.1 \%$ \\
\hline 24 (7.5\%) & $4.9 \%, 11.0 \%$ \\
\hline 97 (30.5\%) & $25.5 \%, 35.9 \%$ \\
\hline 55 (17.3\%) & $13.3 \%, 21.9 \%$ \\
\hline $1(0.3 \%)$ & $0.0 \%, 1.7 \%$ \\
\hline 161 (50.6\%) & $45.0 \%, 56.3 \%$ \\
\hline $3(0.9 \%)$ & $0.2 \%, 2.7 \%$ \\
\hline 40 (12.6\%) & $9.1 \%, 16.7 \%$ \\
\hline 114 (35.8\%) & $30.6 \%, 41.4 \%$ \\
\hline 33 (10.4\%) & $7.3 \%, 14.3 \%$ \\
\hline $2(0.6 \%)$ & $0.1 \%, 2.3 \%$ \\
\hline 127 (39.9\%) & $34.5 \%, 45.6 \%$ \\
\hline 207 (65.1\%) & $59.6 \%, 70.3 \%$ \\
\hline 111 (34.9\%) & $29.7 \%, 40.4 \%$ \\
\hline 296 (93.1\%) & $89.7 \%, 95.6 \%$ \\
\hline 22 (6.9\%) & $4.4 \%, 10.3 \%$ \\
\hline 260 (81.8\%) & $77.1 \%, 85.8 \%$ \\
\hline 58 (18.2\%) & $14.2 \%, 22.9 \%$ \\
\hline 25 (7.9\%) & $5.2 \%, 11.4 \%$ \\
\hline 293 (92.1\%) & $88.6 \%, 94.8 \%$ \\
\hline $15(4.7 \%)$ & $2.7 \%, 7.7 \%$ \\
\hline
\end{tabular}

Delayed or timed cord clamping with COVID-19 Positive Mother Yes

No

Delayed or timed cord clamping with non-COVID Positive Mother

Yes

No

Early baths ( $<4$ hours) with COVID-19 Positive Mother

Yes

No

Early baths ( $<4$ hours) with non-COVID-19 Positive Mother

Yes

No

$15(4.7 \%)$ 
Table 1 (continued)

\begin{tabular}{|c|c|c|}
\hline \multirow[t]{2}{*}{ Overall, n (\%) } & $\mathrm{N}(\%)$ & $95 \% \mathrm{Cl}$ \\
\hline & \multicolumn{2}{|l|}{$318(100 \%)$} \\
\hline Discouraged, but permitted if family strongly desires & $101(31.8 \%)$ & $26.7 \%, 37.2 \%$ \\
\hline Encouraged with precautions & $49(15.4 \%)$ & $11.6 \%, 19.9 \%$ \\
\hline Decisions based on shared decision making on a case-by-case basis & $153(48.1 \%)$ & $42.5 \%, 53.8 \%$ \\
\hline \multicolumn{3}{|c|}{ Approach to expressed breast milk with COVID-19 Positive Mother (check all that apply) } \\
\hline May be given by the mother with precautions & $189(59.4 \%)$ & $53.9 \%, 64.9 \%$ \\
\hline May be given by another caregiver & $245(77.0 \%)$ & $72.0 \%, 81.6 \%$ \\
\hline Discouraged & $3(0.9 \%)$ & $0.2 \%, 2.7 \%$ \\
\hline
\end{tabular}

Approach to testing for an infant delivered by cesarean section with anticipated discharge on day 3 or 4 with a COVID-19 Positive Mother

We generally do not test infants

We do 1 test

We do 2 tests

More than 2 tests

Unsure

Other $^{3}$

Time of first test ( $n=265$; respondents that reported 1, 2, or $2+$ tests)

Before 24 hours

Around 24 hours

Between 24-48 hours

Around 48 hours or after 48 hours

Time of second test ( $n=172$; respondents that reported 2 or $2+$ tests)

Between 24-48 hours

Around 48 hours

After 48 hours

\section{Discharge Processes for Infants with COVID-19 Positive Mothers}

Hearing screening

Have not changed process

Changed process, but occurs during hospitalization

Deferred until after discharge

\section{$31(9.7 \%)$ \\ $93(29.2 \%)$ \\ $169(53.1 \%)$ \\ $3(0.9 \%)$ \\ $19(6.0 \%)$ \\ $3(0.9 \%)$}

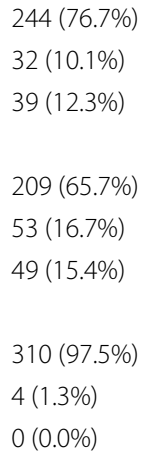

\section{Newborn screen}

Have not changed process

Changed process, but occurs during hospitalization

Deferred until after discharge

\section{Congenital heart disease screen}

Have not changed process

Changed process, but occurs during hospitalization

Deferred until after discharge
$12(4.5 \%)$

$199(75.1 \%)$

$42(15.8 \%)$

$9(3.4 \%)$

$7(4.1 \%)$

$141(82.0 \%)$

$23(13.4 \%)$

$6.7 \%, 13.6 \%$

$24.3 \%, 34.6 \%$

$47.5 \%, 58.7 \%$

$0.2 \%, 2.7 \%$

$3.6 \%, 9.2 \%$

$0.2 \%, 2.7 \%$

$2.4 \%, 7.8 \%$ $69.4 \%, 80.2 \%$ $11.7 \%, 20.8 \%$ $1.6 \%, 6.3 \%$

$1.7 \%, 8.2 \%$ $75.4 \%, 87.4 \%$ $8.7 \%, 19.4 \%$

$71.7 \%, 81.3 \%$

$7.0 \%, 13.9 \%$

$8.9 \%, 16.4 \%$

$60.2 \%, 70.9 \%$

$12.7 \%, 21.2 \%$

$11.6 \%, 19.9 \%$

$95.1 \%, 98.9 \%$

$0.3 \%, 3.2 \%$

$-$

$\begin{array}{ll}297(93.4 \%) & 90.1 \%, 95.9 \% \\ 18(5.7 \%) & 3.4 \%, 8.8 \% \\ 0(0.0 \%) & -- \\ & \\ 297(93.4 \%) & 90.1 \%, 95.9 \% \\ 18(5.7 \%) & 3.4 \%, 8.8 \% \\ 0(0.0 \%) & -- \\ 301(94.7 \%) & 91.6 \%, 96.9 \% \\ 13(4.1 \%) & 2.2 \%, 6.9 \% \\ 0(0.0 \%) & --\end{array}$


Table 1 (continued)

\begin{tabular}{|c|c|c|}
\hline \multirow[t]{2}{*}{ Overall, n (\%) } & $N(\%)$ & $95 \% \mathrm{Cl}$ \\
\hline & \multicolumn{2}{|l|}{$318(100 \%)$} \\
\hline \multicolumn{3}{|l|}{ Red reflex } \\
\hline Have not changed process & $306(96.2 \%)$ & $93.5 \%, 98.0 \%$ \\
\hline Changed process, but occurs during hospitalization & $5(1.6 \%)$ & $0.5 \%, 3.6 \%$ \\
\hline Deferred until after discharge & $3(0.9 \%)$ & $0.2 \%, 2.7 \%$ \\
\hline \multicolumn{3}{|c|}{ Discharge Processes for non-COVID-19 Positive Mother-Infant Dyads } \\
\hline \multicolumn{3}{|c|}{ Timing of Discharge } \\
\hline Timing hasn't really changed & $129(40.6 \%)$ & $35.1 \%, 46.2 \%$ \\
\hline Some dyads are discharged early & $91(28.6 \%)$ & $23.7 \%, 33.9 \%$ \\
\hline Many dyads are discharged early & $73(23.0 \%)$ & $18.4 \%, 28.0 \%$ \\
\hline All dyads discharge early unless a medical contraindication & $24(7.5 \%)$ & $4.9 \%, 11.0 \%$ \\
\hline
\end{tabular}

$P P E$ personal protective equipment, $L \& D$ labor and delivery

'Other maternal testing answers were test based on symptoms OR a scheduled admission for delivery $(n=4)$, test for a scheduled admission $(n=4)$, "based on obstetrical practice" $(n=1)$, and "universal test at 38 weeks" $(n=1)$

${ }^{2}$ Other location of newborn care answers were if mother asymptomatic and desires same room and if mother symptomatic separate rooms $(n=3)$

${ }^{3}$ Other newborn testing answers were "test infant if symptomatic" $(n=1)$, decision made on case by case basis by provider $(n=1)$ or infection control $(n=1)$

hospitals offered universal testing to pregnant women who were anticipated to deliver. More than $95 \%$ of hospitals reported that the pediatric team wore N95 masks, eye protection, gloves and gowns as PPE for vaginal deliveries of COVID-19 positive women. Visitors were restricted to only 1 support person among 98\% of hospitals and $78 \%$ did not offer or require any testing of support persons. Approach to location of newborn care varied substantially; we found that shared decision making was the preferred approach among $50 \%$ of hospitals, separate rooms among $31 \%$, and care provided in the same room among 17\%. Other breastfeeding support practices, including skin-to-skin care, and approaches to direct breastfeeding and provision of expressed mother's milk also showed substantial variation (Table 1). Delayed or timed cord clamping for COVID-19 positive mothers was the approach among $65 \%$ of hospitals, compared to 93\% for non-COVID-19 positive mothers; early bathing of infants born to COVID-19 positive mothers was the approach for $82 \%$ of hospitals, compared to $8 \%$ for infants born to non-COVID-19 positive mothers. With respect to infant testing, 53\% of hospitals performed two tests and $29 \%$ performed one test, while $10 \%$ did not routinely test infants. We found that hearing screening was deferred until after discharge among $12 \%$ and circumcisions were deferred until after discharge among $15 \%$ of U.S. hospitals among infants born to mothers who tested positive for SARS-CoV-2, Finally, discharges occurred early for at least some presumed uninfected dyads among $59 \%$ of U.S. hospitals in our survey.

We found substantial regional variation in several COVID-19 related hospital practices (Table 2). The likelihood that a hospital offered universal testing of women expected to deliver and offering or requiring testing for asymptomatic support persons was highest in the Northeast. Regarding infant testing, hospitals in the South had the highest rate of testing each infant twice. Care in separate rooms and prohibiting and discouraging direct breastfeeding were highest in the South, whereas shared decision making was used most often among hospitals in the West. Early discharge of dyads was reported most frequently among hospitals in the Northeast.

Hospital practices did not vary significantly according to highest level of neonatal care (Additional File A), with the exception of skin-to-skin care in the first hour after birth among mothers with positive tests, where prohibiting or discouraging skin-to-skin care was highest among hospitals providing level 3 and 4 neonatal care (51\%), compared to hospitals with level $1(44 \%)$ and level $2(40 \%)$ neonatal care $(p=0.024)$. Hospital breastfeeding support practices did not vary by Baby-Friendly hospital status (Additional File B).

Finally, among all individual respondents, we considered each individual's personal preferred approach to location of care, and whether this differed from their hospital's approach. Among 433 individual respondents, 174 (40\%) preferred that decisions be based on shared decision making on a case-by-case basis, 128 (30\%) preferred care of dyads in same room with precautions, 105 (24\%) preferred care of dyads in separate rooms, 14 (3\%) had no particular opinion, 8 (2\%) preferred care of dyads in the same room without precautions, and $4(0.9 \%)$ gave other responses. Table 3 shows the concordance of hospital and individual preferred approaches to location of care, 
Table 2 COVID-19 Related Hospital Care Practices Among Mothers with Healthy, Term Infants According to US Region 1,2

\begin{tabular}{|c|c|c|c|c|c|}
\hline Overall, n (\%) & Northeast & Midwest & South & West & $p$-value \\
\hline \multicolumn{6}{|l|}{ Obstetric and Delivery Practices } \\
\hline Testing of Women Anticipated to Deliver & & & & & 0.043 \\
\hline Universal testing & $69(88.5 \%)$ & $51(77.3 \%)$ & $64(67.4 \%)$ & $51(64.6 \%)$ & \\
\hline Testing based on signs and symptoms & $7(9.0 \%)$ & $10(15.2 \%)$ & $27(28.4 \%)$ & $24(30.4 \%)$ & \\
\hline Testing not routinely available for pregnant women & $1(1.3 \%)$ & $1(1.5 \%)$ & $0(0.0 \%)$ & $1(1.3 \%)$ & \\
\hline Other ${ }^{3}$ & $1(1.3 \%)$ & $4(6.1 \%)$ & $3(3.2 \%)$ & $2(2.5 \%)$ & \\
\hline \multicolumn{6}{|l|}{ PPE for COVID-19 positive women delivering vaginally (check all that apply) } \\
\hline N95 & $76(97.4 \%)$ & $61(92.4 \%)$ & $92(96.8 \%)$ & $76(96.2 \%)$ & 0.434 \\
\hline Regular Surgical Mask & $3(3.8 \%)$ & $6(9.1 \%)$ & $3(3.2 \%)$ & $5(6.3 \%)$ & 0.358 \\
\hline Eye protection & $76(97.4 \%)$ & $65(98.5 \%)$ & $92(96.8 \%)$ & $77(97.5 \%)$ & 0.934 \\
\hline Cap & $60(76.9 \%)$ & $36(54.5 \%)$ & $69(72.6 \%)$ & $41(51.9 \%)$ & $<.001$ \\
\hline Gown & $77(98.7 \%)$ & $63(95.5 \%)$ & $93(97.9 \%)$ & $72(91.1 \%)$ & 0.068 \\
\hline Gloves & $78(100.0 \%)$ & $64(97.0 \%)$ & $94(98.9 \%)$ & $74(93.7 \%)$ & 0.055 \\
\hline Support persons for pregnant women on Labor and Delivery & & & & & 0.429 \\
\hline No support persons & $0(0.0 \%)$ & $1(1.5 \%)$ & $1(1.1 \%)$ & $0(0.0 \%)$ & \\
\hline Only 1 support person & $77(98.7 \%)$ & $62(93.9 \%)$ & $93(97.9 \%)$ & $79(100.0 \%)$ & \\
\hline 2 or more support persons & $1(1.3 \%)$ & $2(3.0 \%)$ & $0(0.0 \%)$ & $0(0.0 \%)$ & \\
\hline Approach to testing asymptomatic support persons of COVID positive mothers & & & & & 0.006 \\
\hline Not offered or required & $52(66.7 \%)$ & $58(87.9 \%)$ & $77(81.1 \%)$ & $63(79.7 \%)$ & \\
\hline Offer some testing & $12(15.4 \%)$ & $6(9.1 \%)$ & $10(10.5 \%)$ & $13(16.5 \%)$ & \\
\hline Required & $14(17.9 \%)$ & $2(3.0 \%)$ & $6(6.3 \%)$ & $2(2.5 \%)$ & \\
\hline \multicolumn{6}{|l|}{ Newborn Care Practices } \\
\hline Location of newborn care with COVID-19 Positive Mother & & & & & 0.057 \\
\hline Separate room from mother & $17(21.8 \%)$ & $22(33.3 \%)$ & $40(42.1 \%)$ & $18(22.8 \%)$ & \\
\hline Same room as mother with precautions to maintain separation & $20(25.6 \%)$ & $10(15.2 \%)$ & $13(13.7 \%)$ & $12(15.2 \%)$ & \\
\hline Same room as mother with no precautions & $1(1.3 \%)$ & $0(0.0 \%)$ & $0(0.0 \%)$ & $0(0.0 \%)$ & \\
\hline Decisions based on shared decision making on a case-by-case basis & $38(48.7 \%)$ & $34(51.5 \%)$ & $40(42.1 \%)$ & $49(62.0 \%)$ & \\
\hline Other ${ }^{4}$ & $2(2.6 \%)$ & $0(0.0 \%)$ & $1(1.1 \%)$ & $0(0.0 \%)$ & \\
\hline Skin-to-skin care in first hour after birth with COVID-19 Positive Mother & & & & & 0.169 \\
\hline Prohibited & $10(12.8 \%)$ & $8(12.1 \%)$ & $17(17.9 \%)$ & $5(6.3 \%)$ & \\
\hline Discouraged & $24(30.8 \%)$ & $29(43.9 \%)$ & $34(35.8 \%)$ & $27(34.2 \%)$ & \\
\hline Encouraged with precautions & $10(12.8 \%)$ & $6(9.1 \%)$ & $9(9.5 \%)$ & $8(10.1 \%)$ & \\
\hline Encouraged with no precautions & $0(0.0 \%)$ & $0(0.0 \%)$ & $2(2.1 \%)$ & $0(0.0 \%)$ & \\
\hline Decisions based on shared decision making on a case-by-case basis & $34(43.6 \%)$ & $23(34.8 \%)$ & $33(34.7 \%)$ & $37(46.8 \%)$ & \\
\hline \multicolumn{6}{|l|}{ Other } \\
\hline Delayed or timed cord clamping with COVID-19 Positive Mother & & & & & 0.087 \\
\hline Yes & $57(73.1 \%)$ & $39(59.1 \%)$ & $55(57.9 \%)$ & $56(70.9 \%)$ & \\
\hline No & $21(26.9 \%)$ & $27(40.9 \%)$ & $40(42.1 \%)$ & $23(29.1 \%)$ & \\
\hline Delayed or timed cord clamping with non-COVID Positive Mother & & & & & 0.066 \\
\hline Yes & $73(93.6 \%)$ & $61(92.4 \%)$ & $84(88.4 \%)$ & $78(98.7 \%)$ & \\
\hline No & $5(6.4 \%)$ & $5(7.6 \%)$ & $11(11.6 \%)$ & $1(1.3 \%)$ & \\
\hline Early baths ( $<4$ hours) with COVID-19 Positive Mother & & & & & 0.152 \\
\hline Yes & $60(76.9 \%)$ & $59(89.4 \%)$ & $80(84.2 \%)$ & $61(77.2 \%)$ & \\
\hline No & $18(23.1 \%)$ & $7(10.6 \%)$ & $15(15.8 \%)$ & $18(22.8 \%)$ & \\
\hline Early baths ( $<4$ hours) with non-COVID-19 Positive Mother & & & & & 0.616 \\
\hline Yes & $6(7.7 \%)$ & $5(7.6 \%)$ & $10(10.5 \%)$ & $4(5.1 \%)$ & \\
\hline No & $72(92.3 \%)$ & $61(92.4 \%)$ & $85(89.5 \%)$ & $75(94.9 \%)$ & \\
\hline Approach to direct breastfeeding with COVID-19 Positive Mother & & & & & 0.028 \\
\hline Prohibited & $3(3.8 \%)$ & $4(6.1 \%)$ & $6(6.3 \%)$ & $2(2.5 \%)$ & \\
\hline
\end{tabular}


Table 2 (continued)

\begin{tabular}{|c|c|c|c|c|c|}
\hline Overall, n (\%) & Northeast & Midwest & South & West & $p$-value \\
\hline Discouraged, but permitted if family strongly desires & $15(19.2 \%)$ & $22(33.3 \%)$ & $43(45.3 \%)$ & $21(26.6 \%)$ & \\
\hline Encouraged with precautions & $15(19.2 \%)$ & $8(12.1 \%)$ & $11(11.6 \%)$ & $15(19.0 \%)$ & \\
\hline Decisions based on shared decision making on a case-by-case basis & $45(57.7 \%)$ & $32(48.5 \%)$ & $35(36.8 \%)$ & $41(51.9 \%)$ & \\
\hline \multicolumn{6}{|c|}{ Approach to expressed breast milk with COVID-19 Positive Mother (check all that apply) } \\
\hline May be given by the mother with precautions & $48(61.5 \%)$ & $39(59.1 \%)$ & $52(54.7 \%)$ & $50(63.3 \%)$ & 0.682 \\
\hline May be given by another caregiver & $59(75.6 \%)$ & $56(84.8 \%)$ & $72(75.8 \%)$ & $58(73.4 \%)$ & 0.387 \\
\hline Discouraged & $0(0.0 \%)$ & $1(1.5 \%)$ & $0(0.0 \%)$ & $2(2.5 \%)$ & 0.260 \\
\hline $\begin{array}{l}\text { Approach to testing for an infant delivered by cesarean section with antici- } \\
\text { pated discharge on day } 3 \text { or } 4 \text { with COVID-19 Positive Mother }\end{array}$ & & & & & 0.003 \\
\hline We generally do not test infants & $6(7.7 \%)$ & $8(12.1 \%)$ & $6(6.3 \%)$ & $11(13.9 \%)$ & \\
\hline We do 1 test & $35(44.9 \%)$ & $15(22.7 \%)$ & $20(21.1 \%)$ & $23(29.1 \%)$ & \\
\hline We do 2 tests & $35(44.9 \%)$ & $37(56.1 \%)$ & $63(66.3 \%)$ & $34(43.0 \%)$ & \\
\hline More than 2 tests & $0(0.0 \%)$ & $1(1.5 \%)$ & $1(1.1 \%)$ & $1(1.3 \%)$ & \\
\hline Unsure & $0(0.0 \%)$ & $5(7.6 \%)$ & $4(4.2 \%)$ & $10(12.7 \%)$ & \\
\hline Other ${ }^{5}$ & $2(2.6 \%)$ & $0(0.0 \%)$ & $1(1.1 \%)$ & $0(0.0 \%)$ & \\
\hline Time of first test ( $n=262$; respondents that reported 1,2 , or $2+$ tests) & & & & & 0.041 \\
\hline Before 24 hours & $1(1.4 \%)$ & $1(1.9 \%)$ & $5(6.0 \%)$ & $5(8.6 \%)$ & \\
\hline Around 24 hours & $49(70.0 \%)$ & $37(69.8 \%)$ & $69(82.1 \%)$ & $44(75.9 \%)$ & \\
\hline Between 24-48 hours & $17(24.3 \%)$ & $10(18.9 \%)$ & $7(8.3 \%)$ & $8(13.8 \%)$ & \\
\hline Around 48 hours or after 48 hours & $2(2.8 \%)$ & $5(9.4 \%)$ & $2(2.4 \%)$ & $0(0.0 \%)$ & \\
\hline Time of second test ( $n=171$; respondents that reported 2 or $2+$ tests) & & & & & 0.341 \\
\hline Between 24-48 hours & $1(2.9 \%)$ & $0(0.0 \%)$ & $4(6.3 \%)$ & $2(5.7 \%)$ & \\
\hline Around 48 hours & $29(82.9 \%)$ & $29(76.3 \%)$ & $55(85.9 \%)$ & $28(80.0 \%)$ & \\
\hline After 48 hours & $5(14.3 \%)$ & $9(23.7 \%)$ & $4(6.3 \%)$ & $5(14.3 \%)$ & \\
\hline \multicolumn{6}{|l|}{ Discharge Processes } \\
\hline Hearing screening & & & & & 0.131 \\
\hline Have not changed process & $59(75.6 \%)$ & $51(77.3 \%)$ & $73(76.8 \%)$ & $61(77.2 \%)$ & \\
\hline Changed process, but occurs during hospitalization & $11(14.1 \%)$ & $10(15.2 \%)$ & $4(4.2 \%)$ & $7(8.9 \%)$ & \\
\hline Deferred until after discharge & $8(10.3 \%)$ & $5(7.6 \%)$ & $17(17.9 \%)$ & $9(11.4 \%)$ & \\
\hline Circumcisions & & & & & 0.107 \\
\hline Have not changed process & $50(64.1 \%)$ & $43(65.2 \%)$ & $59(62.1 \%)$ & $57(72.2 \%)$ & \\
\hline Changed process, but occurs during hospitalization & $17(21.8 \%)$ & $16(24.2 \%)$ & $12(12.6 \%)$ & $8(10.1 \%)$ & \\
\hline Deferred until after discharge & $10(12.8 \%)$ & $7(10.6 \%)$ & $21(22.1 \%)$ & $11(13.9 \%)$ & \\
\hline Hepatitis B & & & & & 0.396 \\
\hline Have not changed process & $76(97.4 \%)$ & $65(98.5 \%)$ & $94(98.9 \%)$ & 75 (94.9\%) & \\
\hline Changed process, but occurs during hospitalization & $2(2.6 \%)$ & $0(0.0 \%)$ & $0(0.0 \%)$ & $2(2.5 \%)$ & \\
\hline Deferred until after discharge & $0(0.0 \%)$ & $0(0.0 \%)$ & $0(0.0 \%)$ & $0(0.0 \%)$ & \\
\hline Bilirubin checks & & & & & 0.007 \\
\hline Have not changed process & $67(85.9 \%)$ & $63(95.5 \%)$ & $91(95.8 \%)$ & $76(96.2 \%)$ & \\
\hline Changed process, but occurs during hospitalization & $11(14.1 \%)$ & $3(4.5 \%)$ & $3(3.2 \%)$ & $1(1.3 \%)$ & \\
\hline Deferred until after discharge & $0(0.0 \%)$ & $0(0.0 \%)$ & $0(0.0 \%)$ & $0(0.0 \%)$ & \\
\hline Newborn screen & & & & & 0.231 \\
\hline Have not changed process & $70(89.7 \%)$ & $63(95.5 \%)$ & $89(93.7 \%)$ & 75 (94.9\%) & \\
\hline Changed process, but occurs during hospitalization & $8(10.3 \%)$ & $3(4.5 \%)$ & $5(5.3 \%)$ & $2(2.5 \%)$ & \\
\hline Deferred until after discharge & $0(0.0 \%)$ & $0(0.0 \%)$ & $0(0.0 \%)$ & $0(0.0 \%)$ & \\
\hline Congenital heart disease screen & & & & & 0.450 \\
\hline Have not changed process & $72(92.3 \%)$ & $63(95.5 \%)$ & $92(96.8 \%)$ & $74(93.7 \%)$ & \\
\hline Changed process, but occurs during hospitalization & $6(7.7 \%)$ & $2(3.0 \%)$ & $2(2.1 \%)$ & $3(3.8 \%)$ & \\
\hline Deferred until after discharge & $0(0.0 \%)$ & $0(0.0 \%)$ & $0(0.0 \%)$ & $0(0.0 \%)$ & \\
\hline Red reflex & & & & & 0.323 \\
\hline
\end{tabular}


Table 2 (continued)

\begin{tabular}{|c|c|c|c|c|c|}
\hline Overall, n (\%) & Northeast & Midwest & South & West & $p$-value \\
\hline Have not changed process & $74(94.9 \%)$ & $65(98.5 \%)$ & $91(95.8 \%)$ & $76(96.2 \%)$ & \\
\hline Changed process, but occurs during hospitalization & $3(3.8 \%)$ & $1(1.5 \%)$ & $0(0.0 \%)$ & $1(1.3 \%)$ & \\
\hline Deferred until after discharge & $1(1.3 \%)$ & $0(0.0 \%)$ & $2(2.1 \%)$ & $0(0.0 \%)$ & \\
\hline \multicolumn{6}{|c|}{ Discharge Processes for non-COVID-19 Positive Mother-Infant Dyads } \\
\hline Timing of Discharge & & & & & $<0.001$ \\
\hline Timing hasn't really changed & $17(21.8 \%)$ & $28(42.4 \%)$ & $48(50.5 \%)$ & $36(45.6 \%)$ & \\
\hline Some dyads are discharged early & $20(25.6 \%)$ & $16(24.2 \%)$ & $29(30.5 \%)$ & $26(32.9 \%)$ & \\
\hline Many dyads are discharged early & $29(37.2 \%)$ & $20(30.3 \%)$ & $16(16.8 \%)$ & $8(10.1 \%)$ & \\
\hline All dyads discharge early unless a medical contraindication & $12(15.4 \%)$ & $2(3.0 \%)$ & $2(2.1 \%)$ & $8(10.1 \%)$ & \\
\hline
\end{tabular}

PPE personal protective equipment, $L \& D$ labor and delivery

${ }^{1}$ Chi-square $p$-values shown; Missing answers not shown (this occurred $<1.5 \%$ of all questions)

${ }^{2}$ Northeast includes Maine, New Hampshire, Vermont, Massachusetts, Connecticut, Rhode Island, New York, New Jersey, Pennsylvania, Delaware, Maryland, Virginia, West Virginia, and Washington D.C.; Midwest includes Minnesota, Wisconsin, Michigan, lowa, Illinois, Missouri, Indiana, Ohio, and Kentucky; South includes Arkansas, Tennessee, North Carolina, Louisiana, Mississippi, Alabama, Georgia, and Florida; West includes Washington, Oregon, Idaho, California, Nevada, Utah, Arizona, Hawaii, and Alaska

${ }^{3}$ Other maternal testing answers were test based on symptoms OR a scheduled admission for delivery $(n=4)$, test for a scheduled admission ( $\left.n=4\right)$, "based on obstetrical practice" ( $n=1)$, and "universal test at 38 weeks" $(n=1)$

${ }^{4}$ Other location of newborn care answers were if mother asymptomatic and desires same room and if mother symptomatic separate rooms ( $n=3$ )

${ }^{5}$ Other newborn testing answers were "test infant if symptomatic" $(n=1)$, decision made on case by case basis by provider $(n=1)$ or infection control $(n=1)$

Table 3 Congruence Between Personal Preference and Hospital Approach to Location of Care

\begin{tabular}{|c|c|c|c|c|}
\hline \multirow[t]{2}{*}{ Hospital Approach to Location of Care } & \multicolumn{3}{|c|}{ Personal Preferred Approach to Location of Care ${ }^{2}$} & \multirow{2}{*}{$\begin{array}{l}\text { Total } \\
\text { (sum of row\%) }\end{array}$} \\
\hline & $\begin{array}{l}\text { Separate rooms } \\
\mathrm{N} \text { (row\%) }\end{array}$ & $\begin{array}{l}\text { Same room with } \\
\text { some precautions } \\
\mathrm{N} \text { (row\%) }\end{array}$ & $\begin{array}{l}\text { Shared decision making on } \\
\text { a case-by-case basis } \\
\mathrm{N} \text { (row\%) }\end{array}$ & \\
\hline Separate rooms & $65(48.9 \%)$ & $30(22.6 \%)$ & $38(28.6 \%)$ & $133(33.0 \%)$ \\
\hline Same room with precautions & $4(5.3 \%)$ & $48(64.0 \%)$ & $23(30.7 \%)$ & $75(18.6 \%)$ \\
\hline Shared decision making on a case-by-case basis & $36(18.5 \%)$ & $48(24.6 \%)$ & $111(56.9 \%)$ & $195(48.4 \%)$ \\
\hline Total (column \%) & $105(26.1 \%)$ & $126(31.3 \%)$ & $172(42.5 \%)$ & $403(100 \%)$ \\
\hline
\end{tabular}

${ }^{1}$ Includes 403 individual responses. We excluded 30 respondents (7\%) with: A) no particular personal opinion ( $\left.n=14\right)$, personal preference was same room with no precautions $(n=8)$, personal preference was "other" $(n=4)$, hospital approach was same room with no precautions $(n=1)$, and hospital approach was "other" $(n=3)$.

restricted to the 403 respondents (93\% of sample) that preferred either shared decision making, same room with precautions, or separate rooms and reported that their hospitals used one of these three approaches. Among hospitals with a separation approach, $51 \%$ of respondents from those hospitals preferred an alternative approach. Among hospitals that used a same room with precautions approach, $36 \%$ of respondents from those hospitals preferred an alternative approach, and among hospitals that used a shared-decision making approach, $43 \%$ of respondents from those hospitals preferred an alternative approach.

\section{Discussion}

Physicians from a large sample of hospitals across the U.S. surveyed regarding COVID-19 related newborn care practices from May 17 to 24th demonstrated great variation in several breastfeeding support practices, including location of newborn care, direct breastfeeding, and skin-to-skin care after birth. Adoption of a "shared decision making" approach was most common in the areas of highest variation. We also found that nearly $60 \%$ of hospitals discharged at least some of their presumed uninfected dyads earlier than their usual practice prior to the pandemic. Finally, SARS-CoV-2 testing of mothers, asymptomatic support persons, and infants, as well as approaches to location of care, direct breastfeeding and timing of discharge varied according to US region, but not by highest level of neonatal care or Baby-Friendly Hospital Status.

Hospitals routinely create guidelines or policies with the goal of reducing provider variation and/or optimizing the delivery of standardized, evidenced-based care. Considering the paucity of population-level data that 
could best inform practices for newborn care in the setting of the COVID-19 pandemic in the spring of 2020, it is not surprising that we found wide national variation in several COVID-19 newborn care practices. Similar to our findings, Ahmad et al recently reported variation in maternal testing, location of care, and direct breastfeeding among 368 hospitals assessed by 4 serial surveys from March to August 2020 among U.S. hospitals affiliated with the Mednax network [13]. While we did not perform serial surveys to examine changes over time, our study included nearly all US states, therefore representing a broader range of US hospitals and we additionally examined newborn testing and discharge practices, skinto-skin care, delayed cord clamping and early baths. Variation was likely driven in part by differing guidance by major professional organizations in the spring of 2020 . Our study showed that among several breastfeeding support practices, shared decision making, which was endorsed by the CDC in their statement on April 4, 2020 [6], was the most frequently used hospital approach and most frequent personal preferred approach to location of care among individual survey respondents. Shared decision making occurs when healthcare professionals and patients make decisions together with both parties cognizant of available evidence, the limits of evidence, and uncertainties related to the possible benefits and harms of each options [14]. We speculate that, in the setting of uncertainty regarding the health risks and benefits of these various practices, many hospital teams and individual providers felt comfortable engaging families directly in the process of decision-making.

We also found that some hospital practices were not consistent with professional organization guidelines. Local context may be a driver for this variation. For example, we found that universal testing of women anticipated to deliver was most frequent in Northeastern hospitals, which may have represented increased local testing availability, and the fact that the pandemic was most acute in this region at the time of survey administration. It is also possible that changes in discharge processes, such as timing of circumcisions and hearing screenings, may have been driven by local preferences of hospital personnel or by a need to conserve PPE. Indeed, an audit completed by 359 hospitals conducted by the Vermont Oxford Network in April 2020 revealed shortages in COVID-19 testing and PPE [15].

While some variation based on local context is likely desirable, other types of variation may not be. If differences in hospital practices reflect external circumstances, rather than local hospital factors, variations may lead to inequities in care. For example, we found higher proportions of hospitals prohibiting/discouraging direct breastfeeding and separating mothers and newborns, practices known to negatively impact the establishment of breastfeeding [16], in the South, compared to other U.S. regions. Prior to the pandemic, breastfeeding rates were lowest in the South [17]. Thus, it is possible that adoption of certain COVID-19 related hospital practices may have exacerbated existing regional disparities in breastfeeding.

The majority of hospitals in our sample (59\%) discharged at least some of their presumed uninfected dyads early, a practice that was not recommended by the WHO, AAP, or the CDC. This may be concerning because many important processes occur during the postpartum hospitalization for the mother and infant which may be missed with early discharge. For example, postpartum women are followed for hypertension, bleeding, and wound healing [11]. Newborns are followed for jaundice, weight loss, and sepsis and require a variety of screening procedures [11]. Further, for most dyads, two days or more are needed for the medical team to assess the adequacy of breastfeeding and for trained professionals to assist with breastfeeding $[11,16]$. Early discharge puts motherinfant dyads at risk for a variety of medical complications that may be less well-monitored in the outpatient setting. In addition, access to outpatient medical providers and lactation support was limited in many areas in the early stages of the COVID-19 pandemic, which may have put maternal-infant dyads at even higher risk due to the decreased ability to ensure good follow-up. Our study did not assess the reasons for early discharge. It is possible that families were asking for early discharge because of their concern for contracting COVID-19 in the hospital or their dissatisfaction with visitation restrictions or other hospital procedures. It is also possible that providers may have more readily offered early discharge because of an interest in reducing any unnecessary staff exposures to patients in the hospital setting. Further examination of the rationale for early discharge is needed to best inform public health messaging and avoid potential adverse consequences of early discharge of healthy mother-infant dyads.

We found that the number of support persons for laboring women was restricted to 1 among $98 \%$ of our sample. While the impact of this practice is unclear for laboring mothers, others have reported adverse effects on parents following widespread restrictions of visitors in the neonatal intensive care unit $[18,19]$. Parents experienced negative impacts on bonding, receipt of provider information, and breastfeeding. Further investigation of the impact of COVID-19 restrictions on the number of support persons during labor and delivery and the postpartum hospitalization is needed.

The strengths of our study are inclusion of a large array of hospitals across 46 U.S. states and examination of many perinatal practices at the hospital-level. We also 
used a two-step approach to sample hospitals. We recognize that a survey administered directly to clinical leaders of newborn nurseries across the U.S. would have been preferred, however, we were unable to identify a comprehensive directory of this nature with up-to-date contact information. It is possible that individual respondents may not have had the most up-to-date and accurate information regarding their hospital's current practices. We minimized this possibility by administering the survey over a short time frame and specifically including the dates during which we aimed to assess current hospital practices in our prompt. It is possible that respondents may have reported their personal practices, rather than the hospital's current practice. Additionally, individuals that completed our survey via listservs and social media may not represent individuals at all US hospitals. There could be bias in response based on individuals particularly interested in the topic. Further, not all physicians working in birthing facilities are members of the AAP Sections of Neonatal Perinatal Medicine, Breastfeeding, and Hospital Medicine or the Neonatal Physician Mother Facebook group. Finally, due to the rapidly evolving state of the COVID-19 pandemic, the findings of our survey may not reflect current hospital practices implemented after professional organizations published guidelines after June 2020 [20, 21]. Nonetheless, the implications of hospital practice variation (overall and by region), including the importance of local context, will likely continue to inform on-going determinations of optimal perinatal care approaches.

\section{Conclusion}

Variation in COVID-19 newborn care practices among U.S. hospitals suggests that mother-infant dyads received different care depending on the hospital and region where delivery took place in late spring 2020. As more evidence and population-level data emerges to inform optimal COVID-19 newborn care practices, consistent adoption of these practices will promote health outcomes and reduce practice variation which may lead to improved health equity. The high rate of early discharge (nearly 60\%) among mother-infant dyads presumed to be uninfected is worrisome in light of potential maternal and neonatal complications that may not be detected or treated timely in the outpatient setting. Overall, we believe that the first surge of the COVID-19 pandemic highlighted the need for professional organizations to work together when publishing guidance, development of data repositories to inform new evidence to drive decisions about best practice and leveraging existing regional and national networks of perinatal centers to facilitate rapid adoption of best practices as they emerge.
Abbreviations

WHO: World Health Organization; AAP: American Academy of Pediatrics; CDC: Centers for Disease Control; US: United States; PPE: Personal protective equipment; Cl: Confidence interval.

\section{Supplementary Information}

The online version contains supplementary material available at https://doi. org/10.1186/s12887-022-03129-0.

\section{Additional file 1.}

Additional file 2.

\section{Acknowledgements}

We acknowledge Emma Jacobson and Matthew Meyer from Boston University for their help in organizing data. We acknowledge Lori Feldman-Winter, Chair of the American Academy of Pediatrics Section on Breastfeeding and Barrett Fromme, Chair of the American Academy of Pediatrics Section on Hospital Medicine, for their support in administering the survey through their listservs.

\section{Authors' contributions}

MGP conceptualized and designed the study, designed data collection instruments, drafted the initial manuscript and reviewed and revised the manuscript. $\mathrm{HH}$ and AP designed data collection instruments, organized and collected data, and reviewed and revised the manuscript. SMK analyzed the data and reviewed and revised the manuscript. AG and MG conceptualized and designed the study, designed data collection instruments, and reviewed and revised the manuscript. TCH conceptualized and designed the study, analyzed data, and reviewed and revised the manuscript. MLH conceptualized the study and revised the manuscript. All authors approved the final manuscript as submitted and agree to be accountable for all aspects of the work.

\section{Funding}

W.K. Kellogg Foundation (PI Parker; P0131665). The funder/sponsor did not participate in this work.

\section{Availability of data and materials}

The data set used during the current study are available from the corresponding author on reasonable request

\section{Declarations}

\section{Ethics approval and consent to participate}

This study was approved as an exempt study by the Boston University Medical Campus Institutional Review Board. All methods were carried out in accordance of guidelines for exempt studies at Boston University Medical Campus. Subjects were informed of their participation of the study and participation was voluntary. Written or verbal was not obtained as the survey results from individuals were anonymous per Boston University Medical Campus IRB guidelines.

\section{Consent for publication}

Not applicable

\section{Competing interests}

Conflict of Interest Statements: MGP, AG and MG participated on the executive leadership committees of the American Academy of Pediatrics Section on Breastfeeding, Hospital Medicine, and Neonatal Perinatal Medicine, respectively, during the conduct of this study. MLH was the current chair of the Section on Neonatal Perinatal Medicine during the conduct of the study. These are unpaid positions. MLH was a co-author of the American Academy of Pediatrics statement on initial guidance of care of infants with COVID-19 published on April 2, 2020. MLH and AG were co-authors of the American Academy of Pediatrics statement on interim guidance for care of infants with COVID-19 published on May 21, 2020. HH, AP, SMK, and TCH have no conflicts of interest.

Financial disclosures: The authors have no financial disclosures. 


\begin{abstract}
Author details
'Department of Pediatrics, Boston Medical Center, Boston University School of Medicine, 801 Albany St, Room 2010, Boston, MA 02446, USA. ${ }^{2}$ Department of Pediatrics, Stanford University School of Medicine, Palo Alto, USA. ${ }^{3}$ Division of Neonatology, Boston Children's Hospital, Harvard Medical School, Boston, USA. ${ }^{4}$ Slone Epidemiology Center, Boston University School of Medicine, Boston, USA. ${ }^{5}$ Department of Biostatistics, Boston University School of Public Health, Boston, USA. ${ }^{6}$ Department of Pediatrics, University of Florida Health, University of Florida College of Medicine, Jacksonville, USA. ${ }^{7}$ Department of Neonatology, Beth Israel Deaconess Medical Center, Harvard Medical School, Boston, USA.
\end{abstract}

Received: 8 January 2021 Accepted: 26 October 2021

Published online: 21 January 2022

\section{References}

1. Gupta M, Zupancic JAF, Pursley DM. Caring for Newborns Born to Mothers With COVID-19: More Questions Than Answers. Pediatrics. 2020;146(2). https://doi.org/10.1542/peds.2020-001842.

2. Stuebe A. Should Infants Be Separated from Mothers with COVID-19? First, Do No Harm. Breastfeed Med. 2020;15(5):351-2. https://doi.org/10. 1089/bfm.2020.29153.ams.

3. Q\&A: Pregnancy, Childbirth and COVID-19. Published online March 18 2020. Accessed August 26, 2020. https://www.who.int/emergencies/ diseases/novel-coronavirus-2019/question-and-answers-hub/q-a-detail/ q-a-on-covid-19-pregnancy-and-childbirth

4. Puopolo KMH, Hudak ML, Kimberlin DW, Cummings J. Initial Guidance: Management of Infants Born to Mothers with COVID-19. Published online April 2, 2020. Accessed May 5, 2020. https://services.aap.org/en/pages/ 2019-novel-coronavirus-covid-19-infections/clinical-guidance/faqsmanagement-of-infants-born-to-covid-19-mothers/

5. FAQs: Management of Infants Born to Mothers with Suspected or Confirmed COVID-19. Published online May 21, 2020. Accessed June 5, 2020. https://services.aap.org/en/pages/2019-novel-coronavirus-covid-19-infec tions/clinical-guidance/faqs-management-of-infants-born-to-covid-19mothers/

6. Coronavirus Disease 2019: Consideration for Inpatient Obstetric Healthcare Settings. Centers for Disease Control. Published online April 4, 2020 Accessed May 4, 2020. https://www.cdc.gov/coronavirus/2019-ncov/ hcp/inpatient-obstetric-healthcare-guidance.html

7. Coronavirus Disease 2019 (COVID-19): Considerations for Inpatient Obstetric Healthcare Settings. Published online May 20, 2020. Accessed August 20, 2020. https://www.cdc.gov/coronavirus/2019-ncov/hcp/inpat ient-obstetric-healthcare-guidance.html

8. Yeo KT, Oei JL, De Luca D, et al. Review of guidelines and recommendations from 17 countries highlights the challenges that clinicians face caring for neonates born to mothers with COVID-19. Acta Paediatr. 2020;109(11):2192-207. https://doi.org/10.1111/apa.15495.

9. NeoQIC COVID-19 Response. Published online June 24, 2020. https:// www.neoqicma.org/covid-19

10. COVID-19 in Newborn Care: Initial Lessons From the Pandemic. Accessed August 22, 2020. https://public.vtoxford.org/covid-19-webinar/

11. Practice $A C$ on F and $N$ and $A C$ on O. Guidelines for Perinatal Care. 8th ed; 2017.

12. Baby-Friendly Facilities A-Z and by State. Accessed August 22, 2020 https://www.babyfriendlyusa.org/for-parents/baby-friendly-facil ities-by-state/

13. Ahmad KA, Darcy-Mahoney A, Kelleher AS, Ellsbury DL, Tolia VN, Clark RH. Longitudinal Survey of COVID-19 Burden and Related Policies in U.S. Neonatal Intensive Care Units. Am J Perinatol. 2021;38(01):093-8. https:// doi.org/10.1055/s-0040-1718944.

14. Barry MJ, Edgman-Levitan S. Shared decision making--pinnacle of patient-centered care. N Engl J Med. 2012;366(9):780-1. https://doi.org/ 10.1056/NEJMp1109283.

15. Horbar JD, Edwards EM, Soll RF, Ehret DEY, Zayack D, Hudak ML. COVID-19 and Newborn Care, vol. 5; 2020
16. Section on Breastfeeding. Breastfeeding and the use of human milk. Pediatrics. 2012;129(3):e827-41. https://doi.org/10.1542/peds.2011-3552.

17. Breastfeeding Report Card United States. 2020. https://www.cdc.gov/ breastfeeding/pdf/2020-Breastfeeding-Report-Card-H.pdf. Accessed 20 Aug 2020.

18. Muniraman H, Ali M, Cawley P, et al. Parental perceptions of the impact of neonatal unit visitation policies during COVID-19 pandemic. BMJPO. 2020;4(1):e000899. https://doi.org/10.1136/bmjpo-2020-000899.

19. Darcy Mahoney A, White RD, Velasquez A, Barrett TS, Clark RH, Ahmad KA. Impact of restrictions on parental presence in neonatal intensive care units related to coronavirus disease 2019. J Perinatol. 2020;40(1):36-46. https://doi.org/10.1038/s41372-020-0753-7.

20. COVID-19: Considerations for Inpatient Obstetric Healthcare Settings. Published online December 1, 2020.

21. FAQs: Management of Infants Born to Mothers with Suspected or Confirmed COVID-19. Published online November 19, 2020. Accessed January 5, 2020. https://services.aap.org/en/pages/2019-novel-coron avirus-covid-19-infections/clinical-guidance/fags-management-of-infan ts-born-to-covid-19-mothers/

\section{Publisher's Note}

Springer Nature remains neutral with regard to jurisdictional claims in published maps and institutional affiliations.

Ready to submit your research? Choose BMC and benefit from

- fast, convenient online submission

- thorough peer review by experienced researchers in your field

- rapid publication on acceptance

- support for research data, including large and complex data types

- gold Open Access which fosters wider collaboration and increased citations

- maximum visibility for your research: over $100 \mathrm{M}$ website views per year

At $\mathrm{BMC}$, research is always in progress.

Learn more biomedcentral.com/submissions 